\title{
Erratum to: Beta autoregressive moving average models
}

\author{
Andréa V. Rocha ${ }^{1}$ - Francisco Cribari-Neto ${ }^{2}$
}

Published online: 6 March 2017

(C) Sociedad de Estadística e Investigación Operativa 2017

\section{Erratum to: TEST (2009) 18:529-545 DOI 10.1007/s11749-008-0112-z}

\section{Introduction}

As noted by Thor Pajhede Nielsen (in correspondence exchanged with the second author and in Pajhede 2017), who is employed at the University of Copenhagen but affiliated with the CREATES center at Aarhus University, the score vector and the conditional Fisher information matrix presented in our article are only correct when the model contains no moving average component. We note that the results presented in Section 5 of the paper, which contains an empirical application, are correct since only $\beta \mathrm{AR}$ models are considered.

In what follows, we shall correct the results in the paper. The expressions that correspond to

$$
\frac{\partial \eta_{t}}{\partial \alpha}, \quad \frac{\partial \eta_{t}}{\partial \beta_{l}}, \quad \frac{\partial \eta_{t}}{\partial \varphi_{i}}, \quad \text { and } \quad \frac{\partial \eta_{t}}{\partial \theta_{j}} \text {. }
$$

The online version of the original article can be found under doi:10.1007/s11749-008-0112-z.

$凶 \quad$ Andréa V. Rocha

andrea.rocha@ci.ufpb.br

Francisco Cribari-Neto

cribari@de.ufpe.br

1 Departamento de Computação Científica, Universidade Federal da Paraíba, Mangabeira, João Pessoa, PB 58058-600, Brazil

2 Departamento de Estatística, Universidade Federal de Pernambuco, Cidade Universitária, Recife, PE 50670-901, Brazil 
are in error. The error propagates throughout the paper and renders the following expressions incorrect:

- In what concerns the score vector: the vector $U_{\alpha}(\gamma)$, the matrices $M, P$ and $R$;

- In what concerns Fisher's information matrix: $\mathbb{E}\left(\frac{\partial^{2} \ell}{\partial \alpha^{2}} \mid \mathscr{F}_{t-1}\right), \mathbb{E}\left(\frac{\partial^{2} \ell}{\partial \alpha \partial \phi} \mid \mathscr{F}_{t-1}\right)$, $\mathbb{E}\left(\frac{\partial^{2} \ell}{\partial \alpha \partial \beta} \mid \mathscr{F}_{t-1}\right), \mathbb{E}\left(\frac{\partial^{2} \ell}{\partial \varphi \partial \alpha} \mid \mathscr{F}_{t-1}\right), \mathbb{E}\left(\frac{\partial^{2} \ell}{\partial \theta \partial \alpha} \mid \mathscr{F}_{t-1}\right)$, and matrices $M, P$ and $R ;$

- In what concerns hypothesis testing inference (Section 4): the score vector, Fisher's information matrix $K$, and matrices $X$ and $Z_{t}$.

\section{Computation of $\frac{\partial \eta_{t}}{\partial \alpha}, \frac{\partial \eta_{t}}{\partial \beta_{l}}, \frac{\partial \eta_{t}}{\partial \varphi_{i}}$, and $\frac{\partial \eta_{t}}{\partial \theta_{j}}$}

We shall now correct the expressions for the derivatives in (1). At the outset, recall that our time series model is given by

$$
g\left(\mu_{t}\right)=\eta_{t}=\alpha+x_{t}^{\prime} \beta+\sum_{i=1}^{p} \varphi_{i}\left\{g\left(y_{t-i}\right)-x_{t-i}^{\prime} \beta\right\}+\sum_{j=1}^{q} \theta_{j} r_{t-j}
$$

We arrive at the following recursions:

$$
\begin{aligned}
& \frac{\partial \eta_{t}}{\partial \alpha}=1+\sum_{j=1}^{q} \theta_{j} \frac{\partial r_{t-j}}{\partial \alpha}, \\
& \frac{\partial \eta_{t}}{\partial \beta}=x_{t}^{\prime}-\sum_{i=1}^{p} \varphi_{i} x_{t-i}^{\prime}+\sum_{j=1}^{q} \theta_{j} \frac{\partial r_{t-j}}{\partial \beta}, \\
& \frac{\partial \eta_{t}}{\partial \varphi_{i}}=g\left(y_{t-i}\right)-x_{t-i}^{\prime} \beta+\sum_{j=1}^{q} \theta_{j} \frac{\partial r_{t-j}}{\partial \varphi_{i}}, \quad i=1, \ldots, p,
\end{aligned}
$$

and

$$
\frac{\partial \eta_{t}}{\partial \theta_{l}}=r_{t-l}+\sum_{j=1}^{q} \theta_{j} \frac{\partial r_{t-j}}{\partial \theta_{l}}, \quad l=1, \ldots, q
$$

As in Benjamin et al. (1998), recursions only take place when the model includes moving average components. For autoregressive models, the results in Rocha and Cribari-Neto (2009) are correct. When the model includes moving average dynamics, it is necessary to choose initial values for $\eta_{t}$ and its derivatives. The initial values for $\eta_{t}$ are taken to be $\eta_{t}=g\left(y_{t}\right)$ and the derivatives initial values are set equal to zero, for $t=1, \ldots, q$.

In the next subsections, we shall provide explicit expressions for the relevant derivatives considering the two errors $\left(r_{t}\right)$ mentioned in the paper. 


\subsection{Error $r_{t}=y_{t}-\mu_{t}$}

We begin by noting that

$$
r_{t}=y_{t}-\mu_{t}=y_{t}-g^{-1}\left(\eta_{t}\right)
$$

Let $\lambda$ be a surrogate for $\alpha, \beta, \varphi_{i}$ or $\theta_{j}$. We obtain

$$
\frac{\partial r_{t}}{\partial \lambda}=\frac{\partial r_{t}}{\partial \eta_{t}} \frac{\partial \eta_{t}}{\partial \lambda}
$$

which yields

$$
\frac{\partial r_{t}}{\partial \lambda}=-\frac{1}{g^{\prime}\left(\mu_{t}\right)} \frac{\partial \eta_{t}}{\partial \lambda}
$$

Therefore, we obtain the following recursions for the derivatives of $\eta_{t}$ :

$$
\begin{aligned}
\frac{\partial \eta_{t}}{\partial \alpha} & =1-\sum_{j=1}^{q} \theta_{j} \frac{1}{g^{\prime}\left(\mu_{t-j}\right)} \frac{\partial \eta_{t-j}}{\partial \alpha}, \\
\frac{\partial \eta_{t}}{\partial \beta} & =x_{t}^{\prime}-\sum_{i=1}^{p} \varphi_{i} x_{t-i}^{\prime}-\sum_{j=1}^{q} \theta_{j} \frac{1}{g^{\prime}\left(\mu_{t-j}\right)} \frac{\partial \eta_{t-j}}{\partial \beta}, \\
\frac{\partial \eta_{t}}{\partial \varphi_{i}} & =g\left(y_{t-i}\right)-x_{t-i}^{\prime} \beta-\sum_{j=1}^{q} \theta_{j} \frac{1}{g^{\prime}\left(\mu_{t-j}\right)} \frac{\partial \eta_{t-j}}{\partial \varphi_{i}}, \quad i=1, \ldots, p,
\end{aligned}
$$

and

$$
\frac{\partial \eta_{t}}{\partial \theta_{l}}=y_{t-l}-\mu_{t-l}-\sum_{j=1}^{q} \theta_{j} \frac{1}{g^{\prime}\left(\mu_{t-j}\right)} \frac{\partial \eta_{t-j}}{\partial \theta_{l}}, \quad l=1, \ldots, q
$$

\subsection{Error $r_{t}=g\left(y_{t}\right)-\eta_{t}$}

Here,

$$
r_{t}=g\left(y_{t}\right)-\eta_{t} .
$$

It follows that

$$
\frac{\partial r_{t}}{\partial \lambda}=-\frac{\partial \eta_{t}}{\partial \lambda}
$$


We thus obtain the following recursions for the derivatives of $\eta_{t}$ :

$$
\begin{aligned}
& \frac{\partial \eta_{t}}{\partial \alpha}=1-\sum_{j=1}^{q} \theta_{j} \frac{\partial \eta_{t-j}}{\partial \alpha}, \\
& \frac{\partial \eta_{t}}{\partial \beta}=x_{t}^{\prime}-\sum_{i=1}^{p} \varphi_{i} x_{t-i}^{\prime}-\sum_{j=1}^{q} \theta_{j} \frac{\partial \eta_{t-j}}{\partial \beta}, \\
& \frac{\partial \eta_{t}}{\partial \varphi_{i}}=g\left(y_{t-i}\right)-x_{t-i}^{\prime} \beta-\sum_{j=1}^{q} \theta_{j} \frac{\partial \eta_{t-j}}{\partial \varphi_{i}}, \quad i=1, \ldots, p,
\end{aligned}
$$

and

$$
\frac{\partial \eta_{t}}{\partial \theta_{l}}=g\left(y_{t-l}\right)-\eta_{t-l}-\sum_{j=1}^{q} \theta_{j} \frac{\partial \eta_{t-j}}{\partial \theta_{l}}, \quad l=1, \ldots, q
$$

\section{Remaining expressions}

\subsection{Score vector}

Recall that $m=\max \{p, q\}$. We have

$$
\begin{aligned}
\frac{\partial \ell}{\partial \alpha} & =\phi \sum_{t=m+1}^{n}\left(y_{t}^{*}-\mu_{t}^{*}\right) \frac{1}{g^{\prime}\left(\mu_{t}\right)} \frac{\partial \eta_{t}}{\partial \alpha} \\
& =\phi \sum_{t=m+1}^{n}\left(y_{t}^{*}-\mu_{t}^{*}\right) \frac{1}{g^{\prime}\left(\mu_{t}\right)}\left(1+\sum_{j=1}^{q} \theta_{j} \frac{\partial r_{t-j}}{\partial \alpha}\right) .
\end{aligned}
$$

Let $s$ be an $(n-m)$ row vector whose $i$ th element is given by

$$
\frac{\partial \eta_{i+m}}{\partial \alpha}=1+\sum_{j=1}^{q} \theta_{j} \frac{\partial r_{i+m-j}}{\partial \alpha}
$$

Thus,

$$
U_{\alpha}(\gamma)=\phi s^{\prime} T\left(y^{*}-\mu^{*}\right)
$$

It then follows that

$$
\begin{aligned}
\frac{\partial \ell}{\partial \beta} & =\phi \sum_{t=m+1}^{n}\left(y_{t}^{*}-\mu_{t}^{*}\right) \frac{1}{g^{\prime}\left(\mu_{t}\right)} \frac{\partial \eta_{t}}{\partial \beta} \\
& =\phi \sum_{t=m+1}^{n}\left(y_{t}^{*}-\mu_{t}^{*}\right) \frac{1}{g^{\prime}\left(\mu_{t}\right)}\left(x_{t}^{\prime}-\sum_{i=1}^{p} \varphi_{i} x_{t-i}^{\prime}+\sum_{j=1}^{q} \theta_{j} \frac{\partial r_{t-j}}{\partial \beta}\right) .
\end{aligned}
$$


Let $M$ be the $(n-m) \times k$ matrix whose $i$ th row given is by

$$
\frac{\partial \eta_{i+m}}{\partial \beta}=x_{i+m}^{\prime}-\sum_{l=1}^{p} \varphi_{l} x_{i+m-l}^{\prime}+\sum_{j=1}^{q} \theta_{j} \frac{\partial r_{i+m-j}}{\partial \beta} .
$$

We obtain

$$
U_{\beta}(\gamma)=\phi M^{\prime} T\left(y^{*}-\mu^{*}\right)
$$

Differentiation with respect to $\varphi_{i}, i=1, \ldots, p$, yields

$$
\begin{aligned}
\frac{\partial \ell}{\partial \varphi_{i}} & =\phi \sum_{t=m+1}^{n}\left(y_{t}^{*}-\mu_{t}^{*}\right) \frac{1}{g^{\prime}\left(\mu_{t}\right)} \frac{\partial \eta_{t}}{\partial \varphi_{i}} \\
& =\phi \sum_{t=m+1}^{n}\left(y_{t}^{*}-\mu_{t}^{*}\right) \frac{1}{g^{\prime}\left(\mu_{t}\right)}\left(g\left(y_{t-i}\right)-x_{t-i}^{\prime} \beta+\sum_{j=1}^{q} \theta_{j} \frac{\partial r_{t-j}}{\partial \varphi_{i}}\right) .
\end{aligned}
$$

Thus, if we let $P$ be the $(n-m) \times p$ matrix whose $(i, j)$ th element is given by

$$
\frac{\partial \eta_{i+m}}{\partial \varphi_{j}}=g\left(y_{i+m-j}\right)-x_{i+m-j}^{\prime} \beta+\sum_{l=1}^{q} \theta_{l} \frac{\partial r_{i+m-l}}{\partial \varphi_{j}}
$$

we obtain

$$
U_{\varphi}(\gamma)=\phi P^{\prime} T\left(y^{*}-\mu^{*}\right)
$$

Finally, differentiation with respect to $\theta_{j}, j=1, \ldots, q$, yields

$$
\begin{aligned}
\frac{\partial \ell}{\partial \theta_{j}} & =\phi \sum_{t=m+1}^{n}\left(y_{t}^{*}-\mu_{t}^{*}\right) \frac{1}{g^{\prime}\left(\mu_{t}\right)} \frac{\partial \eta_{t}}{\partial \theta_{j}} \\
& =\phi \sum_{t=m+1}^{n}\left(y_{t}^{*}-\mu_{t}^{*}\right) \frac{1}{g^{\prime}\left(\mu_{t}\right)}\left(r_{t-j}+\sum_{l=1}^{q} \theta_{l} \frac{\partial r_{t-l}}{\partial \theta_{j}}\right) .
\end{aligned}
$$

Let $R$ be the $(n-m) \times q$ matrix whose $(i, j)$ th element is

$$
\frac{\partial \eta_{i+m}}{\partial \theta_{j}}=r_{i+m-j}+\sum_{l=1}^{q} \theta_{l} \frac{\partial r_{i+m-l}}{\partial \theta_{j}}
$$

We obtain

$$
U_{\theta}(\gamma)=\phi R^{\prime} T\left(y^{*}-\mu^{*}\right)
$$




\subsection{Fisher's information matrix}

At the outset, we note that expressions for $M, P$ and $R$ are as in the previous subsection. The remaining expressions are

$$
\begin{aligned}
& \mathbb{E}\left(\frac{\partial^{2} \ell}{\partial \alpha^{2}} \mid \mathscr{F}_{t-1}\right)=-\phi s^{\prime} W s, \quad \mathbb{E}\left(\frac{\partial^{2} \ell}{\partial \alpha \partial \phi} \mid \mathscr{F}_{t-1}\right)=-s^{\prime} T c, \\
& \mathbb{E}\left(\frac{\partial^{2} \ell}{\partial \alpha \partial \beta} \mid \mathscr{F}_{t-1}\right)=-\phi M^{\prime} W s, \quad \mathbb{E}\left(\frac{\partial^{2} \ell}{\partial \varphi \partial \alpha} \mid \mathscr{F}_{t-1}\right)=-\phi P^{\prime} W s,
\end{aligned}
$$

and

$$
\mathbb{E}\left(\frac{\partial^{2} \ell}{\partial \theta \partial \alpha} \mid \mathscr{F}_{t-1}\right)=-\phi R^{\prime} W s
$$

\subsection{Hypothesis testing inference}

The score vector and Fisher's information matrix are as in the previous subsections. The matrix $X$ obtained as

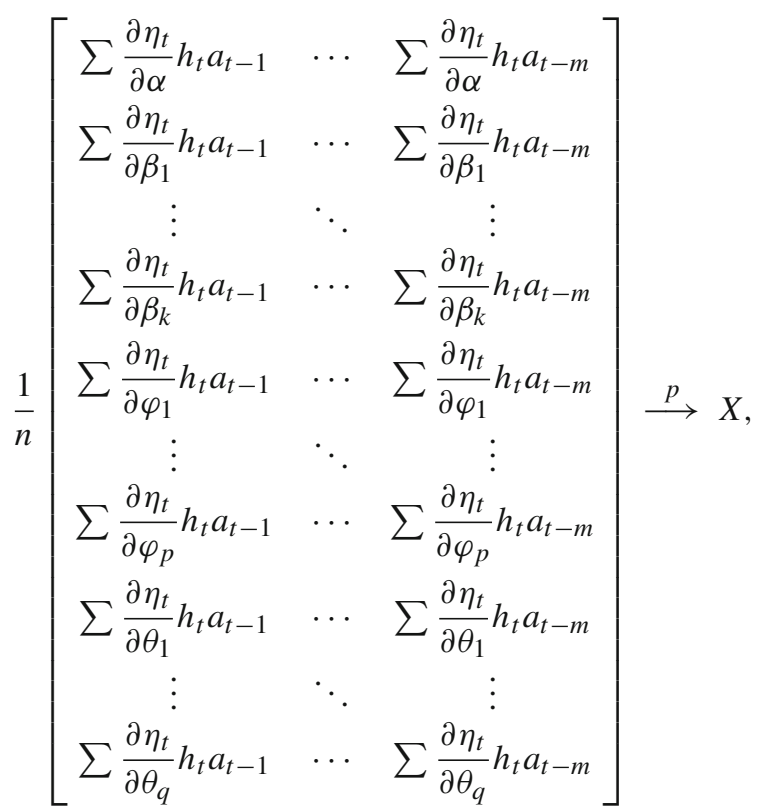

where $\stackrel{p}{\longrightarrow}$ denotes convergence in probability, and

$$
h_{t}=\frac{\left(\psi^{\prime}\left(\mu_{t} \phi\right)+\psi^{\prime}\left(\left(1-\mu_{t}\right) \phi\right)\right)^{1 / 2}}{g^{\prime}\left(\mu_{t}\right)} .
$$


We shall now list conditions that guarantee the existence of the above limiting matrix. We begin by recalling that the standardized score residuals

$$
a_{t}=\frac{y_{t}^{\star}-\mu_{t}^{\star}}{\sqrt{\psi^{\prime}\left(\mu_{t} \phi\right)+\psi^{\prime}\left(\left(1-\mu_{t}\right) \phi\right)}}
$$

satisfy $\mathbb{E}\left(a_{t} \mid \mathscr{F}_{t_{1}}\right)=0$ and $\operatorname{Var}\left(a_{t} \mid \mathscr{F}_{t-1}\right)=1 / \phi$. Thus, the sequence $\left(a_{t}\right)$ is a martingale difference sequence with constant variance. Our goal is to apply Theorem 2.19 of Hall and Heyde (1980). For the reader's convenience, we shall state it here:

Theorem Let $\left\{\mathfrak{X}_{n}, n \geq 1\right\}$ be a sequence of random variables and $\left\{\mathscr{G}_{n}, n \geq 1\right\}$ an increasing sequence of $\sigma$-fields with $\mathfrak{X}_{n}$ measurable with respect to $\mathscr{G}_{n}$ for each $n$. Let $\mathfrak{X}$ be a random variable and c a constant such that $\mathbb{E}|\mathfrak{X}|<\infty$ and $\operatorname{Pr}\left(\left|\mathfrak{X}_{n}\right|>x\right) \leq$ $c \operatorname{Pr}(|\mathfrak{X}|>x)$ for each $x \geq 0$ and $n \geq 1$. Then,

$$
\frac{1}{n} \sum_{i=1}^{n}\left[\mathfrak{X}_{i}-\mathbb{E}\left(\mathfrak{X}_{i} \mid \mathscr{G}_{i-1}\right)\right] \stackrel{p}{\longrightarrow} 0
$$

as $n \rightarrow \infty$.

The above theorem can be applied if following assumptions to hold:

Assumption (A): $\sup _{t} h_{t}<\infty$.

Assumption (B): $\sup _{t}\left|\frac{\partial \eta_{t}}{\partial \lambda}\right|<\infty$, where $\lambda$ is a surrogate for $\alpha, \beta$ or $\varphi$. Notice that for such parameters the derivative $\frac{\partial \eta_{t}}{\partial \lambda}$ is deterministic.

Therefore, under Assumptions (A) and (B) the sequences $\mathfrak{X}_{t}^{j}=\frac{\partial \eta_{t}}{\partial \lambda} h_{t} a_{t-j}$, for $j=1, \ldots, m$, satisfy the assumptions of the above theorem for $\mathscr{G}_{t}=\mathscr{F}_{t-j}$. Indeed, we must only show that there exists a positive integrable random variable $A$ such that $\operatorname{Pr}\left(\left|a_{t}\right|>x\right) \leq \operatorname{Pr}(A>x)$ for each $x \geq 0$ and each $t \geq 1$, as the remaining conditions are trivially satisfied. Note that we have the following unconditional moments: $\mathbb{E}\left(a_{t}\right)=$ 0 and $\operatorname{Var}\left(a_{t}\right)=1 / \phi$. Thus, applying Chebyshev's inequality we obtain

$$
\operatorname{Pr}\left(\left|a_{t}\right|>x\right) \leq \frac{1}{\phi x^{2}}
$$

Let $F$ be a distribution function such that $F(x)=0$ if $x<1 / \sqrt{\phi}$ and $F(x)=$ $1-1 /\left(\phi x^{2}\right)$ if $x \geq 1 / \sqrt{\phi}$, and let $A$ be a random variable with distribution function $F$. It is easy to show that $A$ is integrable. Since $A$ does not depend on $t$, we have proved our claim.

Furthermore, for $i \geq j$,

$$
\mathbb{E}\left(\mathfrak{X}_{t}^{j} \mid \mathscr{F}_{t-j-1}\right)=\frac{\partial \eta_{t}}{\partial \lambda} h_{t} \mathbb{E}\left(a_{t-j} \mid \mathscr{F}_{t-j-1}\right)=0 .
$$

Thus, it follows from the theorem that 


$$
\frac{1}{n} \sum \frac{\partial \eta_{t}}{\partial \lambda} h_{t} a_{t-j} \stackrel{p}{\longrightarrow} 0
$$

This shows that the elements of the matrix $X$ related to the parameters $\alpha, \beta$ and $\varphi$ are all equal to zero. That follows from the fact that the only random element is $a_{t}$, which has mean zero, and the law of large numbers holds for such variables.

We shall now move to the parameters related to $\theta$. We shall need another assumption:

Assumption (C): For each $k=1, \ldots, q$, there exists a square integrable random variable $\mathfrak{T}_{k}$ and a constant $c>0$ such that $\operatorname{Pr}\left(\left|\frac{\partial \eta_{t}}{\partial \theta_{k}}\right|>x\right) \leq c \operatorname{Pr}\left(\left|\mathfrak{T}_{k}\right|>x\right)$.

We have that, under Assumptions (A) and (C), the sequences $\mathfrak{X}_{t}^{j, k}=\frac{\partial \eta_{t}}{\partial \theta_{k}} h_{t} a_{t-j}$, for $j=1, \ldots, m$, satisfy the assumptions of the theorem for $\mathscr{G}_{t}=\mathscr{F}_{t-j}$. Indeed, let $A$ be the random variable used in the previous case and note that $A$ is square integrable. Since $h_{t}$ is deterministic and bounded by Assumption (A), we may disregard this term in our computations in order to keep notation simple. Thus, we must find a positive integrable random variable $B$ and a constant $d>0$ such that $\operatorname{Pr}\left(\left|\frac{\partial \eta_{t}}{\partial \theta_{k}} a_{t-j}\right|>x\right) \leq$ $d \operatorname{Pr}(B>x)$. To that end, note that

$$
\begin{aligned}
\operatorname{Pr}\left(\left|\frac{\partial \eta_{t}}{\partial \theta_{k}} a_{t-j}\right|>x\right) & \leq \operatorname{Pr}\left(\left(\frac{\partial \eta_{t}}{\partial \theta_{k}}\right)^{2}+a_{t-j}^{2}>2 x\right) \\
& \leq \operatorname{Pr}\left(\left(\frac{\partial \eta_{t}}{\partial \theta_{k}}\right)^{2}>x\right)+\operatorname{Pr}\left(a_{t-j}^{2}>x\right) \\
& \leq c \operatorname{Pr}\left(\mathfrak{T}_{j}^{2}>x\right)+\operatorname{Pr}\left(A^{2}>x\right) \\
& \leq(1+c) \operatorname{Pr}\left(\max \left\{\mathfrak{T}_{j}^{2}, A^{2}\right\}>x\right) .
\end{aligned}
$$

Thus, it suffices to take $B=\max \left\{\mathfrak{T}_{j}^{2}, A^{2}\right\}$ and $d=1+c$. Since both $\mathfrak{T}_{j}$ and $A$ are square integrable, it follows that $B$ is an integrable random variable. This shows that our claim holds.

We may also consider the following alternative assumption (to avoid requiring the square integrability of $\mathfrak{T}_{j}$ ):

Assumption ('C'): For each $k=1, \ldots, q$ and each $j=1, \ldots, m$, there exists an integrable random variable $\mathfrak{S}_{k, j}$ and a constant $c>0$ such that $\operatorname{Pr}\left(\left|\frac{\partial \eta_{t}}{\partial \theta_{k}} a_{t-j}\right|>x\right) \leq$ $c \operatorname{Pr}\left(\left|\mathfrak{S}_{k, m}\right|>x\right)$.

Assumption ( $\mathrm{C}^{\prime}$ ) is a direct assumption on the conditions of the theorem.

Therefore, under Assumptions (A) and (C) or (A) and (C'), we may apply the theorem and conclude that for each $k=1, \ldots, q$ and each $j=1, \ldots, m$, the sequence

$$
\frac{1}{n} \sum \frac{\partial \eta_{t}}{\partial \theta_{k}} h_{t} a_{t-j}
$$


has a (possibly nonzero) limit in probability. Thus, under Assumptions (A), (B) and (C) or (A), (B) and (C'), the matrix $X$ exists and is given by

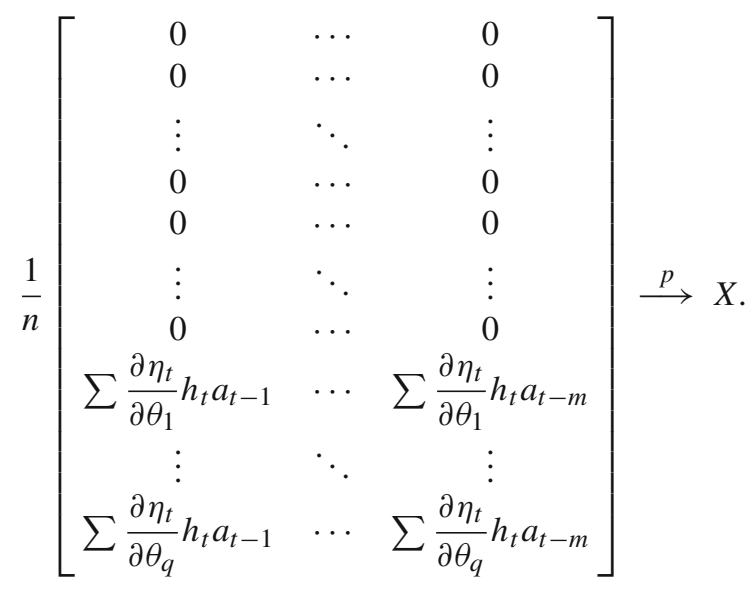

Finally,

$$
Z_{t}=\left(\begin{array}{c}
\frac{\partial \eta_{t}}{\partial \alpha} \\
\frac{\partial \eta_{t}}{\partial \beta_{1}} \\
\vdots \\
\frac{\partial \eta_{t}}{\partial \beta_{k}} \\
\frac{\partial \eta_{t}}{\partial \varphi_{1}} \\
\vdots \\
\frac{\partial \eta_{t}}{\partial \varphi_{p}} \\
\frac{\partial \eta_{t}}{\partial \theta_{1}} \\
\vdots \\
\frac{\partial \eta_{t}}{\partial \theta_{q}}
\end{array}\right)=\left(\begin{array}{c}
1+\sum_{j=1}^{q} \theta_{j} \frac{\partial r_{t-j}}{\partial \alpha} \\
x_{t, 1}-\sum_{i=1}^{p} \varphi_{i} x_{t-i, 1}+\sum_{j=1}^{q} \theta_{j} \frac{\partial r_{t-j}}{\partial \beta_{1}} \\
\vdots \\
x_{t, k}-\sum_{i=1}^{p} \varphi_{i} x_{t-i, k}+\sum_{j=1}^{q} \theta_{j} \frac{\partial r_{t-j}}{\partial \beta_{k}} \\
g\left(y_{t-1}\right)-x_{t-1}^{\prime} \beta+\sum_{j=1}^{q} \theta_{j} \frac{\partial r_{t-j}}{\partial \varphi_{1}} \\
g\left(y_{t-p}\right)-x_{t-p}^{\prime} \beta+\sum_{j=1}^{q} \theta_{j} \frac{\partial r_{t-j}}{\partial \varphi_{p}} \\
r_{t-1}+\sum_{j=1}^{q} \theta_{j} \frac{\partial r_{t-j}}{\partial \theta_{1}} \\
\vdots \\
r_{t-q}+\sum_{j=1}^{q} \theta_{j} \frac{\partial r_{t-j}}{\partial \theta_{q}}
\end{array}\right)
$$

\section{References}

Benjamin MA, Rigby RA, Stasinopoulos MD (1998) Fitting non-Gaussian time series models. In: Payne $\mathrm{R}$, Green P (eds) COMPSTAT proceedings in computational statistics. Physica-Verlag, Heidelberg, pp 191-196

Hall P, Heyde CC (1980) Martingale limit theory and its applications. Academic Press, New York Pajhede T (2017) A conditionally beta distributed time-series model with application to monthly US corporate default rates. Working paper 17-01. Department of Economics, University of Copenhagen Rocha AV, Cribari-Neto F (2009) Beta autoregressive moving average models. Test 18:529-545 